\title{
Cobalt Salophen Complex Supported on Magnetic Nanoparticles as an Efficient Reusable Catalyst for Oxidation of Benzylic Alcohols
}

\author{
MOZHGAN AFSHARI ${ }^{1 *},{\text { MARYAM GORJIZADEH }{ }^{1}, \text { SIMIN NAZARI }}^{2}$ \\ and MOHAMMAD NASEH ${ }^{3}$
}

\begin{abstract}
1Department of Chemistry, Shoushtar Branch, Islamic Azad University, Shoushtar, Iran. ${ }^{2}$ Department of Chemistry, Sousangerd Branch, Islamic Azad University, Sousangerd, Iran.

${ }^{3}$ Department of Chemistry, Dezful Branch, Islamic Azad University, Dezful, Iran.

${ }^{*}$ Corresponding author E-mail: mozhganafshari@yahoo.com
\end{abstract}

http://dx.doi.org/10.13005/ojc/290431

(Received: October 15, 2013; Accepted: December 02, 2013)

\begin{abstract}
A novel and general method has been developed for oxidation of benzylic alcohols using magnetic nanoparticles immobilized salophen $\mathrm{Co}(\mathrm{II})$ as an efficient and recyclable catalyst. The structural and magnetic properties of catalyst are identified by transmission electron microscopy (TEM), vibrating sample magnetometer (VSM) FT-IR and XRD instruments. Nanocatalyst can be easily recovered by a magnetic field and reused for subsequent reactions for at least 5 times with less deterioration in catalytic activity.
\end{abstract}

Key words: Cobalt ferrite, oxidation, Magnetic nanoparticles, Surface functionalization, Cobalt salophen complex.

\section{INTRODUCTION}

Transition metal Schiff base complexes have attracted much interest in the field of catalysis because of their reversible bonding oxygen ability and high activities in the oxidation reactions ${ }^{1-7}$. However, these catalysts are generally homogeneous and are not easily recoverable after oxidation. Also, the work up procedure is not convenient and generates a lot of waste. One of the solutions of these problems is the immobilization of the catalyst on the solid support. Several insoluble solid materials either organic polymers or inorganic porous have been frequently applied as heterogeneous catalytic support for these complexes ${ }^{8-10}$. Inorganic matrices show some advantages over organic supports such as high thermal, chemical and mechanical stability. However, due to the diffusion of substrates and products through the pores of the support materials, a substantial decrease in reaction rate is frequently observed compared to the homogeneous system ${ }^{11-14}$. Thus, it is worthwhile to consider new materials as support for these homogenous catalysts. 
Nowadays, magnetic nanoparticles (MNPs) have attracted increasing interest as promising alternatives for the immobilization of homogeneous catalysts because of their unique properties including a large surface-to-volume ratio, magnetism, low toxicity, biocompatibility and their potential applications in various fields ${ }^{15-20}$. In this study, we wish to present a novel method for the immobilization of a Co (II) salophen complex on imidazole functionalized silica coated cobalt ferrite nanoparticles (Co (salophen)-imid-Si@SiMNPs). The catalytic activity and reusability of this composite in the oxidation of benzylic alcohols was investigated.

\section{EXPERIMENTAL}

\section{General}

Chemical materials were purchased from the Merck Chemical Company in high purity. Cobalt ferrite MNPs were prepared using the procedure reported by Maaz et al. ${ }^{21}$. Silica coated $\mathrm{CoFe}_{2} \mathrm{O}_{4}$ nanoparticles (Si-MNPs) were made by using sol-gel method ${ }^{22}$. X-ray diffraction (XRD) patterns were recorded with a Philips X-ray diffractometer (Model PW1840). FT-IR spectra were obtained using BOMEM MB-Series 1998 FT-IR spectrometer. Magnetic properties of all nanoparticles were measured with a vibrating sample magnetometer (VSM, Meghnatis Daghigh Kavir Company, Iran) at room temperature. NMR spectra were recorded in $\mathrm{CDCl}_{3}$ on a Bruker Advanced DPX $400 \mathrm{MHz}$ spectrometer.

\section{Synthesis of Co (salophen)-imid-Si@Si-MNPs}

A schematic representation for the synthesis of Co (II) salophen complex anchored on imidazole functionalized silica coated cobalt ferrite nanoparticles is shown in Scheme 1. In the first step, imidazole functionalized silica coated magnetic nanoparticles (imid-Si@ Si-MNPs) were achieved by using the previously reported method ${ }^{23}$.

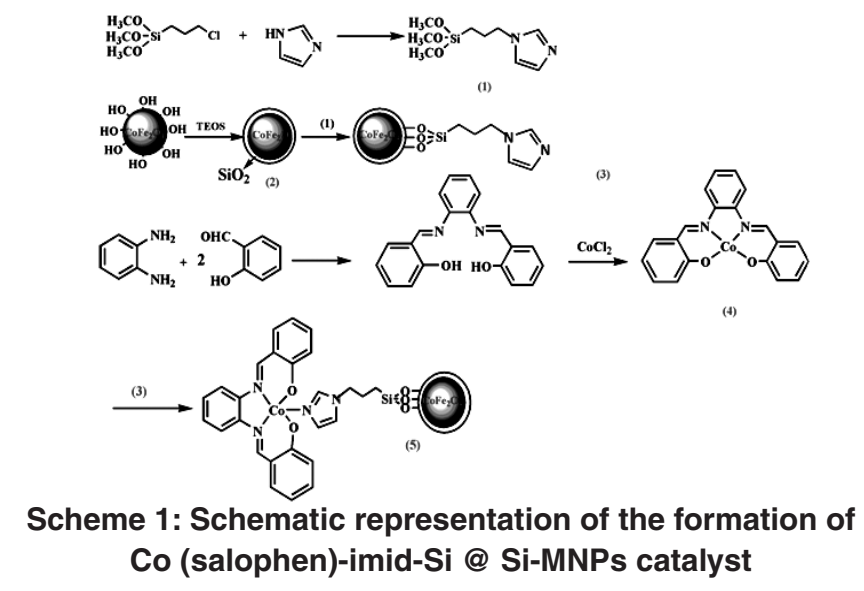

Co (II) complexes of Schiff base ligands have been synthesized by the reaction of $\mathrm{CoCl}_{2}$ in ethanolic solutions with the Schiff bases obtained by the condensation of 1, 2 -phenylenediamine ( 2 $\mathrm{mmol}, 0.216 \mathrm{~g}$ ) with salicylaldehyde $(4 \mathrm{mmol}, 0.43$ $\mathrm{ml})$. Then Co (II) salophen complex was dissolved to 1, 2 dichloroethane and imid-Si@ Si-MNPs (0.75 g) were dispersed to it and the whole mixture was refluxed for 2 days. After that, the expected final product, Co(salophen)-imid-Si @ Si-MNPs , was separated by magnetic decantation and washed with 1,2 dichloroethane, methanol and chloroform until the washings were colorless and left to dry in a desiccator.

\section{Catalytic Studies}

In a typical run, a $25 \mathrm{~mL}$ flask was charged with catalyst $(100 \mathrm{mg})$, acetonitrile $(5 \mathrm{~mL})$, alcohol (1 $\mathrm{mmol})$, and $\mathrm{H}_{2} \mathrm{O}_{2}(3 \mathrm{mmol})$. This mixture was heated in an oil bath at $70{ }^{\circ} \mathrm{C}$ and the progress of the reaction was monitored by TLC. At the end of the reaction, the catalyst was simply collected using a magnetic bar and the reaction mixture was then transferred out of the flask. The decanted solution was purified on 
a silica-gel plate or a silica-gel column to obtain the pure product. The structures of the products were established on the basis of their spectral analysis (IR, 1H NMR).

\section{RESULTS AND DISCUSION}

\section{Characterization of Co (salophen)-imid-Si@} Si-MNPs

The catalyst was synthesized by a procedure that shown in Scheme 1, and characterized by various techniques.
The FT-IR spectrum of heterogenised cobalt salophen shows the expected principal bands for the free schiff base complex. The $\mathrm{C}-\mathrm{H}$ deformation band is visible at $1470 \mathrm{~cm}^{-1}$ and the band at 1611 $\mathrm{cm}^{-1}$ was assigned to $-\mathrm{C}=\mathrm{N}$ stretching vibration of the immine group of the ligand that were not in the imidazole functionalized silica coated cobalt ferrite. The other bands at $589 \mathrm{~cm}^{-1}, 802 \mathrm{~cm}^{-1}$ and $1088 \mathrm{~cm}^{-1}$ ascribed to Fe-O stretching absorption, asymmetrical and symmetrical vibrations of the SiO-Si bond respectively (see Fig. 1) ${ }^{24-30}$.

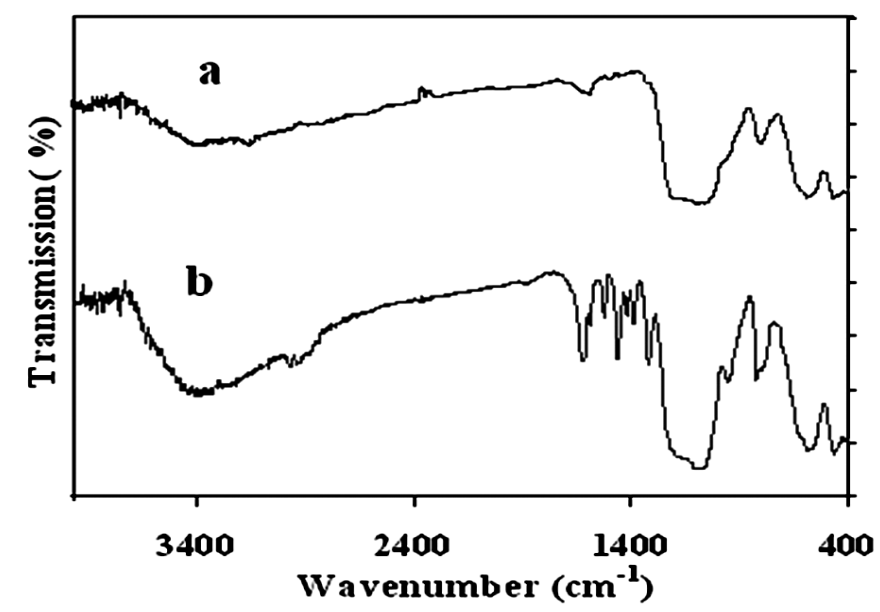

Fig. 1: FT-IR spectra of (a) Si-imid@ Si-MNPs and (b) Co(salophen) -imid-Si @ Si-MNPs

XRD measurements of Co(salophen)-imid-Si @ SiMNPs exhibit diffraction peaks corresponding to the cubic reverse spinel structure of $\mathrm{CoFe}_{2} \mathrm{O}_{4}$ (JCPDS PDF \#221086), while the silica layered systems show an additional broad band at 2 $\theta=20-30$ (Fig. $2 b$ and 2c) for the amorphous silica. The average crystallite size of the Co(salophen)-imid-Si @ Si-MNPs was calculated to be $\sim 28 \mathrm{~nm}$ by Debye-Scherer formula for the (311) reflection.

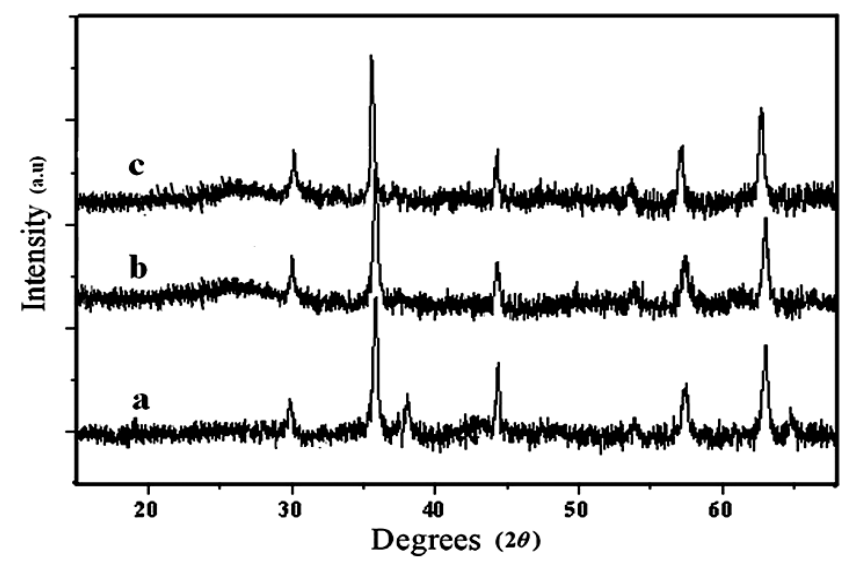

Fig. 2: XRD pattern of (a) MNPs, (b) Si-MNPs and (c) Co(salophen) -imid-Si @ Si-MNPs 
The TEM micrograph of the nanoparticles is shown in Fig.3. TEM image indicated that most of the prepared nanoparticles are spherical shaped and have size less than $30 \mathrm{~nm}$ which show a close agreement with the values calculated by XRD analysis. Interestingly, the magnetic core is visible as a dark spot inside the bright spherical $\mathrm{SiO}_{2}$ thin shell in the TEM image of Co(salophen)-imid-Si @ Si-MNPs sample.

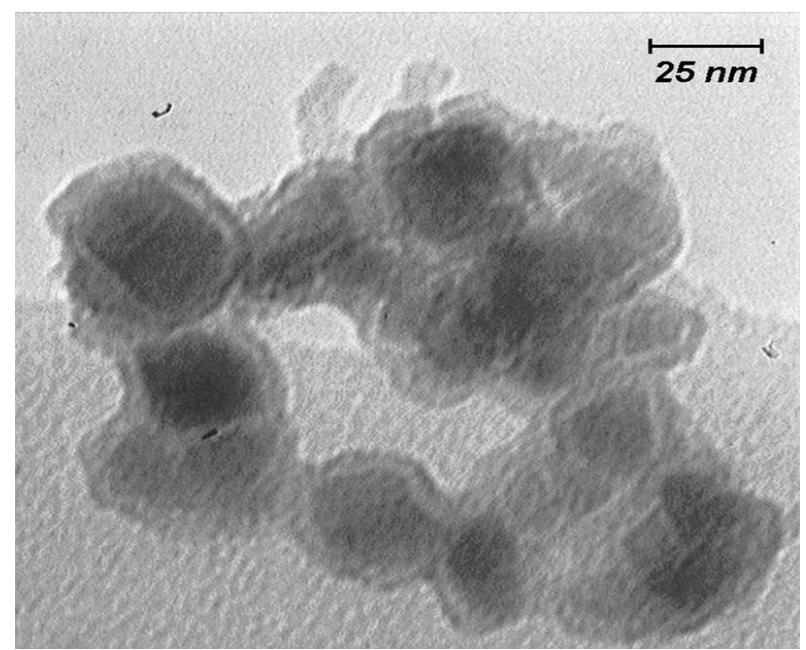

Fig. 3: TEM image of Co(salophen) -imid-Si @ Si-MNPs

The magnetic properties of the silica coated cobalt ferrite (Si-MNPs) and Co (salophen)-imid-Si @ Si-MNPs were studied by a vibrating sample magnetometer at $300 \mathrm{~K}$. The saturation magnetization $\left(M_{s}\right)$, remanence magnetization $\left(M_{r}\right)$ and coercivity $\left(\mathrm{H}_{\mathrm{c}}\right)$ values of these compounds are given in Table 1. As clearly shown, the saturation magnetization of coated sample is slightly less than that of uncoated sample, whereas the coercivity of coated sample is larger than that of uncoated one. This is probably due to the formation of non-crystalline silica on the surface of $\mathrm{CoFe}_{2} \mathrm{O}_{4}$ nanoparticle as well as the restriction of domain wall motion by the magnetic dilution effect of inert silica.

Table 1: Magnetic property of the as-fabricated nanoparticles

\begin{tabular}{llll}
\hline $\mathbf{M}_{\mathbf{s}}(\mathbf{e m u} / \mathbf{g})$ & $\mathbf{M}_{\mathbf{r}}(\mathbf{e m u} / \mathbf{g})$ & $\mathbf{H}_{\mathbf{c}}(\mathbf{O e})$ & compound \\
\hline & & & \\
59.49 & 24.63 & 796.1 & MNPs \\
38.93 & 16.40 & 861.05 & Si-MNPs \\
16.39 & 6.20 & 900 & Co(salophen)- imid -Si@ Si-MNPs \\
\hline
\end{tabular}

As can be seen from thermogravimetric analysis (TGA) the catalyst shows a good thermal stability $\left(\sim 176^{\circ} \mathrm{C}\right)$, with a weight loss at temperature between $173-753^{\circ} \mathrm{C}$, which is characteristic of chemisorbed materials and confirmed that the organic group is covalently bound on the surface of Si-MNPs (Fig. 4). On the basis of the TGA an organic group loading at ca. $0.44 \mathrm{mmol} \mathrm{g}^{-1}$ is obtained. Moreover, the amount of excess cobalt, in addition of $\mathrm{Fe} / \mathrm{Co}$ ratio in cobalt ferrite of the catalyst, determined by ICP-AES, was $0.3 \mathrm{mmol} \mathrm{g}^{-1}$. This is another proof for the fact that Co (II) salophen complex was covalently supported on imidazole functionalized silica coated $\mathrm{CoFe}_{2} \mathrm{O}_{4}$ nanopaticles.

\section{Alcohols}

In order to optimize the reaction conditions for the oxidation of benzylic alcohols various experiments were carried out at $70^{\circ} \mathrm{C}$ using 1-phenylethanol in the presence of heterogenised cobalt salophen as catalyst and $\mathrm{H}_{2} \mathrm{O}_{2}$ as an oxidant. 


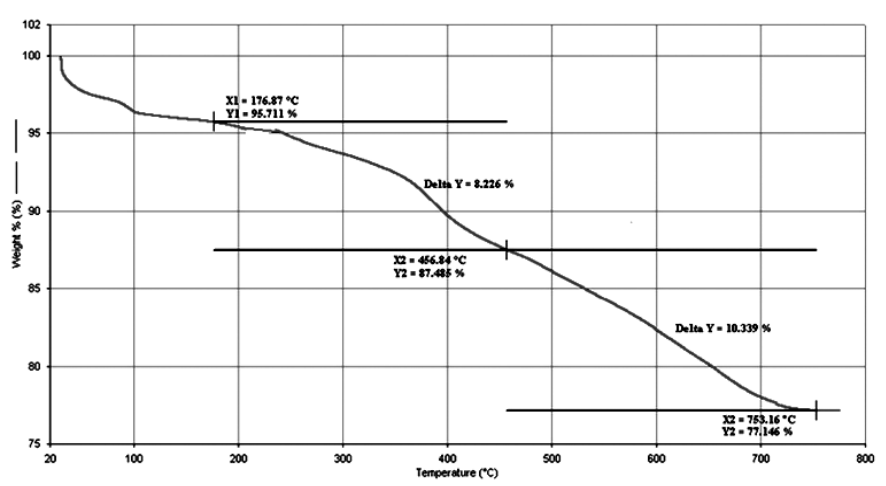

Fig. 4: TGA of Co(salophen)-imid-Si @ Si-MNPs

To optimize the catalyst requirement, the amount of catalyst was changed between 25 and 200 $\mathrm{mg}$ per $1 \mathrm{mmol} 1$-phenylethanol alcohol. Increase in the yield of acetophenone was observed when the amount of catalyst was increased from 25 to 100 $\mathrm{mg}$ but the yield remained almost same with further increment of catalyst amount up to $200 \mathrm{mg}$. So an optimum of $100 \mathrm{mg}$ catalyst in the reaction mixture is ideal for achieving the best yield. In addition, in the absence of catalyst, the reaction was found to be very slow and was not complete even after $8 \mathrm{~h}$. We have also accomplished the oxidation of 1-phenylethanol in the different solvents such as n-hexane, acetone, toluene, cyclohexan, chloroform, dichloromethane and also acetonitrile. Among the solvents examined, we found acetonitrile to be the best for this protocol due to high yield and good dispersibility of the catalyst in this media. Replacing $\mathrm{H}_{2} \mathrm{O}_{2}$ by t-BuOOH, $\mathrm{NaIO}_{4}$ or $\mathrm{H}_{2} \mathrm{O}_{2}$ / Urea (UHP) cause to decrease the obtained total oxidation yields, so $\mathrm{H}_{2} \mathrm{O}_{2}$ was selected as a best source of oxygen for this system.

At optimum conditions we observed that when a heterogeneous mixture of this alcohol and Co(salophen) -imid-Si @ Si-MNPs was heated at 70 ${ }^{\circ} \mathrm{C}$ in acetonitrile, acetophenone was formed as the only product in $94 \%$ yield after $1.5 \mathrm{~h}$.

The obtained preliminary results for the oxidation of 1-phenylethanol were encouraging and to extend the scope of this heterogeneous catalytic system, the oxidation of further alcohols was carried out under the optimized conditions. The given results in Table 2 show that this procedure was clean and all the alcohols were converted into the corresponding carbonyl compounds in moderate to excellent yields without over-oxidation to carboxylic acids.

Interesting electronic effect seemed to exist for these benzylic systems. For example, p-nitrobenzyl alcohol (Table 2, entry 9) required a longer reaction time and gave a decreased yield compared to more electron-rich benzylic substrates. For investigating the chemoselectivity, a mixture of 1-phenylethanol and 2-phenylethanol was subjected under optimized condition in the presence of $\mathrm{Co}$ (salophen) -imid$\mathrm{Si} @$ Si-MNPs. Result indicated that the former was oxidized to acetophenone in $94 \%$ yield while 2-phenylethanol was recovered completely. Furthermore, this reaction is highly selective for vicinal diols in oxidizing only the secondary hydroxy group $\alpha$ to the benzene ring (Table 2, entries 15). Therefore, it is a method of choice for the oxidation of benzylic $\mathrm{OH}$ in the presence of non-benzylic $\mathrm{OH}$.

To evaluate the stability and level of reusability of the catalyst, we conducted experiments of oxidiation of 1-phenylethanol using the recycled Co (salophen)-imid-Si@ Si-MNPs catalyst. After the completion of the first reaction, the catalyst was concentrated on the sidewall of the reaction vessel using an external magnet and the solution was removed by decantation and the left used catalyst was washed with $\mathrm{CH}_{3} \mathrm{CN}$. A new reaction was then conducted with fresh reactants under similar conditions. It was found that the developed catalyst could be used at least five times without any change in reactivity (Table 2, entries 16). Compared with the homogeneous and heterogeneous same catalysts our novel catalyst has shown much more superiority. 
Table 2: Oxidation of alcohols Co (salophen)-imid-Si@ Si-MNPs catalyst ${ }^{\mathrm{a}}$

\begin{tabular}{|c|c|c|c|c|}
\hline Entry & Alochol & Product & Time (h) & Yield $^{\mathrm{b}}(\%)$ \\
\hline 1 & & & 2 & 80 \\
\hline 2 & & & 1.5 & 94 \\
\hline 3 & & & 1.5 & 88 \\
\hline 4 & & & 1.5 & 94 \\
\hline 5 & & & 2 & 80 \\
\hline 6 & & & 1.5 & 95 \\
\hline 7 & & & 2 & 94 \\
\hline 8 & & & 4 & 70 \\
\hline 9 & & & 2 & 65 \\
\hline 10 & & & 1.5 & 70 \\
\hline 11 & & & 1.5 & 94 \\
\hline 12 & & & 1.5 & 95 \\
\hline 13 & & & 2 & 80 \\
\hline 14 & & & 2 & 80 \\
\hline 15 & & & 2 & 90 \\
\hline $16^{c}$ & & & 1.5 & 88 \\
\hline
\end{tabular}

aReaction conditions: allcohol $(1 \mathrm{mmol}), \mathrm{H}_{2} \mathrm{O}_{2}(3 \mathrm{mmol})$, catalyst $(100 \mathrm{mg})$, acetonitrile $(5 \mathrm{ml}), \mathrm{T}=70^{\circ} \mathrm{C}$,

'Isolated yields, ccatalyst : Fifth recycled catalyst 
The observed high activity of the catalyst can be attributed to the nanosized character of the support which increases surface area for more interaction with substrates $26-28$.

\section{CONCLUSION}

In summary the cobalt salophen complex has been supported on the surface-modified $\mathrm{CoFe}_{2} \mathrm{O}_{4}$ magnetic. The synthesized catalyst was confirmed by XRD, FT-IR, TGA, TEM, ICP-AES, and VSM techniques. This nanocatalyst was employed as an efficient catalyst for the oxidation of some benzylic alcohols with hydrogen peroxide. Moreover, the immobilized catalyst could be easily recovered by simple magnetic decantation and reused at least five times without significant loss of activity.

\section{ACKNOWLEDGMENTS}

Authors are thankful to Research Council of Shoushtar Branch, Islamic Azad University, Shoushtar, Iran, for providing financial support for this project.

\section{REFERENCES}

1. E.N. Jacobsen, W. Zhang, M.L. Guler, J. Am. Chem. Soc. 113: 6703 (1991).

2. W. Zhang, J.L. Loebach, S.R. Wilson, E.N. Jacobsen, J. Am. Chem. Soc. 112: 2801 (1990)

3. M. Salavati-Niasari, M. Shakouri-Arani, F. Davar, Microporous Mesoporous Mater. 116: 77 (2008).

4. M. Salavati-Niasari, Microporous Mesoporous Mater. 92: 173 (2006).

5. M. Salavati-Niasari, Microporous Mesoporous Mater. 95, 248 (2006).

6. K.J. Balkus Jr., A.K. Khanmamedova, K.M. Dixon, F. Bedioui, Appl. Catal. A Gen.143: 159 (1996).

7. M.R. Maurya, S.J.J. Titinchi, S. Chand, J. Mol. Catal. A Chem. 214: 257 (2004).

8. S. Bhunia, S. Koner, Polyhedron, 30: 1857 (2011).

9. B. Qi, X. H. Lu, S.Y. Fang, J. Lei, Y.L. Dong, D. Zhou, Q.H. Xia, J. Mol. Catal. A 334, 44, (2011).

10. G. Grivani, S. Tangestaninejad, A.R. Halili, Inorg. Chem. Commun. 10, 914, (2007).

11. P. Ferreira, I.S. Goncalves, F. E. Kuhn, A.D. Lopes, M.A. Martins, M. Pillinger, A. Pina, J. Rocha, C.C. Romao, A.M. Santos, T.M. Santos, A.A. Valente, Eur. J. Inorg. Chem. 2263 (2000)

12. C.D. Nunes, A. A. Valente, M. Pillinger, A.C. Fernandes, C.C. Romao, I. Rocha, I.S. Goncalves, J. Mater. Chem. 12: 1735, ( 2002)
13. M. Jia, A. Seifert, W.R. Thiel, Chem. Mater. 15: 2174 (2003)

14. A. Sakthivel, J. Zhao, G.R. Sieber, M. Hanzlik, A.S.T. Chiang, F.E. Kuhn, Appl. Catal. A. $281267 \quad$ (2005)

15. A. Jutand, S. Negri, A. Principaud, Eur. J. Org. Chem. 631 (2005).

16. S. Cacchi, E. Morera, G. Ortar, Synthesis, 320 (1986).

17. M. Alami, F. Ferri, G. Linstrumelle, Tetrahedron Lett. 34: 6403 (1993).

18. M. Sevilla, T. Valdés-Solis, P. Tartaj, A.B. Fuertes, J. Colloid Interf. Sci. 340: 230 (2009).

19. M. Mahmoudi, S. Sant, B. Wang, S. Laurent, T. Sen, Adv. Drug Deliver. Rev. 63: 24 (2011).

20. V. Polshettiwar, R.S. Varma, Green Chem. 12 : 743 (2010).

21. K. Maaz, A. Mumtaz, S.K. Hasanain, A. Ceylan, J. Magn. Magn. Mater. 308: 289 (2007)

22. Y.H. Deng, C.C. Wang, J.H. Hu, W.L. Yang, S.K. Fu, Colloids Surf. A 262: 87 (2005).

23. M. Kooti, M. Afshari, Mater. Res. Bull. 47: 3473 (2012).

24. Om Prakash, S.A. Iqbal and G. Jacob, Orient J. Chem., 29(3): 1079-1084 (2013).

25. Neeti Rathore and Bal Krishan, Orient J. Chem., 29(3): 1001-1008 (2013).

26. M. Ma, Y. Zhang, W. Yu, H.Y. Shen, H. Zhang, N. Gu, Colloids Surf. A 212, 219, (2003)

27. H. Ono, T. Katsumata, Appl. Phys. Lett.78: 
1832 (2001)

28. F. Rajabi, B. Karimi, J. Mol. Catal. A Chem. 232: 95 (2005).

29. H. Golchibian, S. E. Babaei, Chin. J. Catal.,
31: 615 (2010).

30. N. Gunasekaran, P. Jerome, S. W. Ng, E.R.T. Tiekink, R. Karvembu, J. Mol. Catal. A Chem. 353: 156 (2012). 